\title{
«Expliquem l'Albufera»:
}

\section{transformar una salida de campo en un proyecto interdisciplinar}

\section{«We explain the Albufera»: transforming a field trip into an interdisciplinary project}

\author{
Enric Ortega-Torres \\ Unitat d'Educació, Florida Universitària, Catarroja (València) \\ eortega@florida-uni.es \\ Vicent Moncholí Pons \\ Departament de ciències, Florida Secundària, Catarroja (València) \\ vmoncholi@florida-uni.es
}

RESUMEN • Se expone el proceso seguido por un equipo de docentes de secundaria con el objetivo de cambiar la metodología de trabajo en el aula mediante el diseño y aplicación de una secuencia de aprendizaje definida a partir del aprendizaje basado en retos que integre de manera interdisciplinar los contenidos de Biología y Geografía en segundo de la ESO. Mediante la reflexión didáctica compartida, organizada y continuada se transforma una salida de campo tradicional en un nuevo proyecto de trabajo que se desarrolla a lo largo de un trimestre completo. Los resultados muestran la nueva secuencia de actividades creada, la percepción de los estudiantes y familias y los beneficios en la implicación del alumnado. La conclusión pone de manifiesto el potencial transformador del trabajo en equipo del cuerpo docente.

PALABRAS CLAVE: ABP; Educación Secundaria; Salidas; Codocencia; Interdisciplinariedad.

ABSTRACT - The article explains the process followed by a team of secondary school teachers with the objective of changing the methodology of science learning through the design and application of a learning sequence defined from challenge-based learning that integrates in an interdisciplinary way the contents of biology and geography in the second year of secondary education. Through shared, organized, and continuous didactic reflection, a traditional field trip is transformed into a new interdisciplinary work project that takes place over a full term. The results show the new sequence of activities created, the perception of the students and families and the benefits in the involvement of the students. The conclusion highlights the transformative potential of teachers' teamwork.

KEYWORDS: PBL; Secondary School; Field trip; Co-teaching; Interdisciplinarity.

Recepción: febrero 2020 • Aceptación: febrero 2021 • Publicación: junio 2021 


\section{INTRODUCCIÓN}

La reflexión sobre la práctica es una acción cotidiana que la profesión docente requiere con el fin de encontrar mejoras que pueden integrarse en las actuaciones de aula (Zavala, 2006). Si esta reflexión puede llevarse a cabo en equipo, su potencial transformador crece sustancialmente. Facilitar que un equipo de docentes pueda definir desde la propia reflexión el conjunto de cambios que van a estructurar una actuación didáctica es una de las recomendaciones antiguas que forman parte de las prácticas de codocencia descritas por Cook y Friend (1995) entre otros. En este sentido, entendemos por práctica de codocencia los modos de enseñanza cooperativa desarrollados por dos o más docentes, tal y como la definen Beamish, Bryer y Davies (2006). En este sentido, la actuación que se describe en este artículo se enmarca en una experiencia de investigación-acción donde la coenseñanza está presente en la fase de coordinación, consenso de objetivos y ejecución en paralelo en diferentes grupos de la misma secuencia didáctica, aspectos vertebradores de la codocencia, según autores como Chanmugam y Gerlach (2013) o Conderman y Hedin (2012).

A partir de una reflexión compartida por un equipo de cuatro docentes de secundaria, dos de Ciencias Naturales y otros dos de Ciencias Sociales, estructurada a partir de reuniones de una hora integradas en el horario de todos ellos, fue cómo se ideó, planificó y ejecutó la primera edición del proyecto «Expliquem l'Albufera», que a día de hoy ya cuenta con seis ediciones de éxito.

La necesidad de cambio en las metodologías de enseñanza de las ciencias es una prioridad en las aulas de secundaria; el informe ENCIENDE (COSCE, 2011) expone la importancia de transformar metodologías de enseñanza transmisivas en nuevas metodologías más reflexivas y participativas. Esta necesidad propició la decisión del claustro del centro educativo de diseñar espacios de cuatro horas semanales, en el horario de segundo de la ESO, que a partir de la metodología de aprendizaje basado en proyectos pudieran trabajar de modo interdisciplinar los objetivos de aprendizaje del currículo de Ciencias Naturales y de Ciencias Sociales. Esta intención planteó la necesidad de repensar de nuevo las programaciones de dichas disciplinas.

Entre las diferentes decisiones que había que tomar, el equipo de docentes planteó la importancia de mantener lo que ya funcionaba bien en la trayectoria de las programaciones de las asignaturas implicadas y trataron de integrarlo en los nuevos proyectos pendientes de diseñar. El equipo de docentes coincidió en considerar la «tradicional» salida de campo al Parque Natural de la Albufera como una de las acciones que mejor resultado mostraba en el interés del alumnado. No debía perderse el papel didáctico que ofrecía dicha salida de campo y su potencial favorecedor de actitudes y valores hacia la ciencia (Pedrinaci, 2012). Desde la intención de mantener esta salida como elemento clave en la asimilación de contenidos científicos, se inició un proceso de adaptación para convertirla en un proceso de aprendizaje basado en problemas/retos, tal y como Pedrinaci (2012) propone.

El enfoque que facilitaba el nexo entre los requisitos del currículo de Biología y Geografía e Historia para el diseño del nuevo proyecto se basó en la mirada CTSA y el aprendizaje fuera del aula como elementos clave (Cantó, Hurtado y Vilches, 2013) en el diseńo de la nueva secuencia didáctica.

\section{SECUENCIA DIDÁCTICA}

A partir del conocimiento sobre la situación global respecto a las dificultades para generar interés en el alumnado de secundaria en el aprendizaje de las ciencias, se estableció como prioritario plantear actuaciones que involucrasen al alumnado en actividades que promovieran una indagación real dentro y fuera del aula de ciencias (Britner y Pajares, 2006). Además, se inició la transformación buscando sostener la práctica sobre el aprendizaje social que promueve el trabajo colaborativo siguiendo las sugerencias planteadas por Schunk y Pajares (2002) para el aprendizaje de las ciencias. 
El resultado de la secuencia didáctica diseñada se estructuró siguiendo las bases de la metodología de aprendizaje basado en retos. Se partió de la idea fundamental que comparten todas las versiones que secuencian el conjunto de actividades que configura un proyecto: aprendizaje vinculado a un propósito (Domènech-Casal, 2019) que promueve una participación activa del alumnado a través de la necesidad de uso de habilidades cognitivas de nivel alto (HOTS) para alcanzar mayor profundidad en el aprendizaje (Trujillo, 2016). Siguiendo con la descripción de las características esenciales de las secuencias de ABP descrita por el Buck Institut for Educations (Larmer, Mergendoller y Boss, 2015), podemos describir esta secuencia del siguiente modo (tabla 1):

Tabla 1.

Elementos clave del proyecto

\begin{tabular}{|c|c|}
\hline \multicolumn{2}{|c|}{ Elementos clave del diseño de proyectos (Buck Institut for Educations, 2015) } \\
\hline Problema o pregunta inicial & $\begin{array}{l}\text { El reto planteado es el de construir un centro de interpretación del Parque Natural de } \\
\text { la Albufera en el centro educativo para ayudar al alumnado de primaria de las escuelas } \\
\text { de la localidad a estudiar sus características. }\end{array}$ \\
\hline Indagación sostenida en el tiempo & $\begin{array}{l}\text { El diseño del proyecto se estructura en fases diferenciadas y conectadas entre sí y } \\
\text { facilita la indagación iterada que permite profundizar en el tema. No puede iniciarse } \\
\text { un paso sin haber finalizado el anterior. }\end{array}$ \\
\hline Nivel de autenticidad & $\begin{array}{l}\text { La propuesta de reto se presenta por los propios alumnos de primaria en un encuentro } \\
\text { inicial donde estos solicitan al alumnado de secundaria que se les ayude a estudiar la } \\
\text { Albufera. La proximidad del municipio al parque natural y la posibilidad de diseñar } \\
\text { desde el inicio el centro de interpretación y el formato de visitas guiadas potencian } \\
\text { la autenticidad del proceso y los productos intelectuales generados en el proyecto. }\end{array}$ \\
\hline Empoderamiento del alumnado & $\begin{array}{l}\text { El alumnado se convierte en diseńador, constructor y guía del centro de interpreta- } \\
\text { ción. Transforman su rol, que pasa de ser el de estudiantes de ciencias a expertos en el } \\
\text { parque natural, lo que ayuda a otros a mejorar su conocimiento. }\end{array}$ \\
\hline Reflexión & $\begin{array}{l}\text { La conexión existente entre una acción y la siguiente potencia la necesidad de reflexio- } \\
\text { nar sobre lo aprendido en cada momento. La necesidad de sintetizar la información } \\
\text { con el fin de mostrarla y organizarla para que pueda ser entendida por los futuros } \\
\text { visitantes promueve la reflexión metacognitiva. }\end{array}$ \\
\hline Evaluación formativa & $\begin{array}{l}\text { Cada etapa del proyecto conlleva un tipo de producto entregable que requiere fee- } \\
d b a c k \text { del resto de compañeros y del equipo de docentes. Esta retroalimentación para } \\
\text { la mejora permite al alumnado mejorar sus producciones gracias a la información } \\
\text { recibida. }\end{array}$ \\
\hline Producto público & $\begin{array}{l}\text { El centro de interpretación elaborado queda abierto al público para las visitas de los } \\
\text { estudiantes de los centros de primaria que se realizan con el acompañamiento de } \\
\text { los estudiantes-guías durante el mes siguiente a la inauguración. Además, el centro } \\
\text { sigue abierto durante la visita de los estudiantes de intercambio y para la reunión de } \\
\text { familias del trimestre. }\end{array}$ \\
\hline
\end{tabular}

Con estas características la secuencia ocupaba el segundo trimestre del curso (10 semanas/40 sesiones) y quedaba estructurada siguiendo la siguiente cronología de sesiones:

1. Disparador del proyecto: Visita de los representantes (delegados y tutores) de los grupos de tercero de Primaria de los centros de primaria del municipio para solicitar ayuda al alumnado de segundo de la ESO con el fin de estudiar la Albufera.

2. Inicio del trabajo en grupos base: A partir de la configuración de los grupos de trabajo (grupos heterogéneos propuestos por el equipo de tutores) el equipo de docentes diseña un dosier de investigación cooperativo para conocer los cimientos temáticos del proyecto desde las disciplinas implicadas. Las actividades que integran el dosier se muestran en la tabla 2. 
Tabla 2.

Actividades que componen el dossier de investigación y sus objetivos didácticos

\begin{tabular}{|l|l|}
\hline \multicolumn{1}{|c|}{ Actividad } & \multicolumn{1}{c|}{ Objetivos didácticos } \\
\hline Espacios naturales en Europa & $\begin{array}{l}\text { Conocer el significado de espacio natural } \\
\text { Conocer ejemplos de espacios naturales en Europa }\end{array}$ \\
\hline Parque natural... ¿protegido? & $\begin{array}{l}\text { Estudiar la evolución histórica de la Albufera } \\
\text { Investigar las necesidades de protección del Parque Natural de la Albufera }\end{array}$ \\
\hline El territorio protegido & $\begin{array}{l}\text { Comparar las dimensiones de los diferentes espacios protegidos en la Comu- } \\
\text { nidad Valenciana }\end{array}$ \\
\hline Poema: ruta gastronómica & $\begin{array}{l}\text { Comprender el texto de un poema sobre la Albufera } \\
\text { Mostrar relación entre literatura y entorno geográfico }\end{array}$ \\
\hline Laguna, marjal, albufera. ¿Qué sabes? & Diferenciar conceptos geográficos \\
\hline $\begin{array}{l}\text { Flora y fauna de la Albufera } \\
\text { de Valencia }\end{array}$ & $\begin{array}{l}\text { Conocer la flora y fauna característica de la Albufera } \\
\text { Promover interés por la diversidad del parque natural }\end{array}$ \\
\hline «Pirates de la Marjal» & $\begin{array}{l}\text { Comprensión e interpretación de un relato sobre la Albufera } \\
\text { Valorar las conexiones literarias con el entorno natural }\end{array}$ \\
\hline Qué es un centro de interpretación & Investigar acerca de centros de interpretación \\
\hline
\end{tabular}

3. Trabajo en grupos de expertos: Siguiendo la técnica cooperativa del puzle de Aronson (Aronson y Bridgeman, 1996), a cada uno de los alumnos pertenecientes al grupo base inicial se le asigna un rol diferente para que formen cinco grupos de expertos según sus afinidades e intereses. En este momento del proyecto se realizan tareas específicas del rol de experto escogido. La descripción de la tipología de actividades de cada rol se expone en la tabla 3.

Tabla 3.

Tipología de actividades según el grupo de expertos

\begin{tabular}{|l|l|}
\hline \multicolumn{1}{|c|}{ Grupo de expertos } & \multicolumn{1}{c|}{ Objetivos de la tipología de actividades } \\
\hline Cañas y Barro & $\begin{array}{l}\text { Investigar sobre la flora característica del parque natural. Especial atención a la paja del arroz } \\
\text { y sus usos }\end{array}$ \\
\hline Samarucs & Investigar la fauna característica del parque natural \\
\hline Agua & Investigar las tradiciones de pesca del parque natural \\
\hline Tierra & $\begin{array}{l}\text { Investigar la evolución del parque en cuanto a sus dimensiones y las dificultades de sosteni- } \\
\text { bilidad del entorno }\end{array}$ \\
\hline Tourist-Info & Conocer las actividades culturales que se realizan en el parque natural \\
\hline
\end{tabular}

Dentro de esta fase, además de las actividades propias de cada grupo de expertos, se invita a profesionales externos conocedores del parque natural (biólogos, agricultores, restauradores...) al centro para que realicen encuentros con los diferentes grupos de expertos para propiciar el interés y mostrar referentes cercanos que se vinculen al rol asignado, siguiendo la estrategia propuesta por Zimmerman (2000) para mejorar la autoeficacia del alumnado mediante el acercamiento a modelos referentes.

4. Salida al Parque Natural de la Albufera: En este momento es cuando se organiza un itinerario didáctico para visitar el parque natural. La salida de campo que ya se realizaba se transforma y adapta a la secuencia didáctica diseñada para el proyecto. En esta ocasión el alumnado realiza una modalidad de salida diferente según el grupo de expertos en el que esté trabajando, cada uno hace una ruta y unas actividades diferentes resumidas en la tabla 4. 
Tabla 4.

Tipología de actividades según el grupo de expertos

\begin{tabular}{|l|l|}
\hline Cañas y Barro & Ruta por la Devesa. Identificación de flora \\
\hline Samarucs & Visita a la piscifactoría y avistamiento de aves \\
\hline Agua & Visita a la lonja de pescadores y a la Comunidad de Pescadores de El Palmar \\
\hline Tierra & $\begin{array}{l}\text { Itinerario centrado en construcciones, paisaje y riego y cultivo del arroz. Explicación y visita } \\
\text { al Tancat de la Pipa a pie del campo }\end{array}$ \\
\hline Tourist-Info & $\begin{array}{l}\text { Encuentro con profesionales que organizan actividades dentro del parque. Restauración } \\
\text { (Restaurante Casa Pepe) y Empresa Visit Albufera, atención al visitante, paseos en barca, } \\
\text { alquiler de bicicletas... }\end{array}$ \\
\hline
\end{tabular}

Para finalizar la salida, con un objetivo más lúdico se reúnen de nuevo todos los grupos y se realiza un paseo en barca por la Albufera con un guía local.

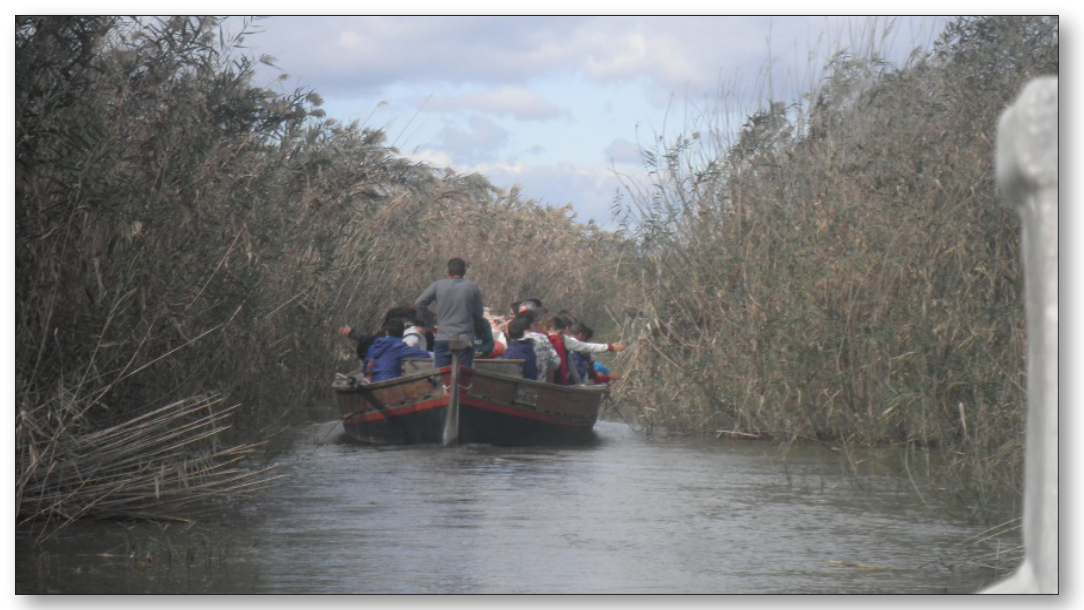

Fig. 1. Momento del paseo en barca.

5. Speed dating entre expertos: Tras la salida de campo realizada al parque natural se organiza una dinámica en el aula para propiciar el cruce de aprendizajes generado a través de las diferentes modalidades de expertos. La dinámica consiste en estructurar las dos horas de sesión de proyecto de modo que cada alumno debe encontrar al menos dos alumnos de cada grupo de expertos diferentes al propio y realizar una entrevista recíproca. Se ha diseñado previamente una hoja de recogida de información para que cada estudiante pueda ordenar toda la información compartida. Cada 15 minutos debe cambiarse de "pareja» y realizar una nueva entrevista.

6. Preparación del centro de interpretación: El alumnado vuelve a la configuración inicial de grupos base y cada experto explica al resto de compañeros en qué ha consistido su trabajo. En este momento se inicia la planificación del producto final del proyecto. El objetivo es preparar un producto expositivo (mural, póster, tríptico, vídeo, experimentos, juegos) que se complemente con el resto de los grupos y forme parte del centro de interpretación de la Albufera. En este proceso de diseño el alumnado de segundo de la ESO debe tener en cuenta que el centro de interpretación va dirigido al alumnado de tercero de Primaria, por lo tanto, debe adaptarse a su realidad. 
En esta etapa del proyecto se realizan sesiones de intercambio de información entre grupos para que todo el alumnado sea consciente del conjunto de productos que van a formar el centro de interpretación y para que proporcionen feedback unos a otros con el fin de mejorar la calidad final de los productos expositivos.

El centro de interpretación formado ocupa diferentes espacios del centro educativo:

- Laboratorio de ciencias: para aportar una mirada científico-experimental de la Albufera, en el laboratorio se estudia el estado del agua, curiosidades sobre el tipo de arroz y el almidón que cada variedad contiene. Además de otros experimentos sobre el suelo y la vegetación.

- Exposición principal: ocupa el hall principal del centro y mediante paneles explicativos y un recorrido didáctico se explica la fauna existente en la Albufera, su flora, así como también la interacción humana y cómo afecta esta a los seres vivos.

- Jardin de paja: situado en la entrada principal del centro, en el patio se ha elaborado un pequeño jardín-laberinto con balas de paja de arroz. El alumnado conoce la problemática de la quema de la paja del arroz y otras posibilidades de uso.



Fig. 2. Visita al jardín de paja del alumnado de primaria.

7. Inauguración del centro y visitas. Una vez finalizado el montaje del centro se invita a los diferentes centros de primaria para que puedan realizar sus visitas guiadas. Cada grupo base se convierte en un equipo guía de los grupos clase de tercero de Primaria y a lo largo de cada visita explica el contenido de la exposición y responde a las preguntas planteadas.

Las visitas finalizan el día de la reunión de final de trimestre con las familias (aproximadamente 300 personas). A lo largo de esa tarde el alumnado también ejerce de guía para explicar el centro de interpretación a las familias. 


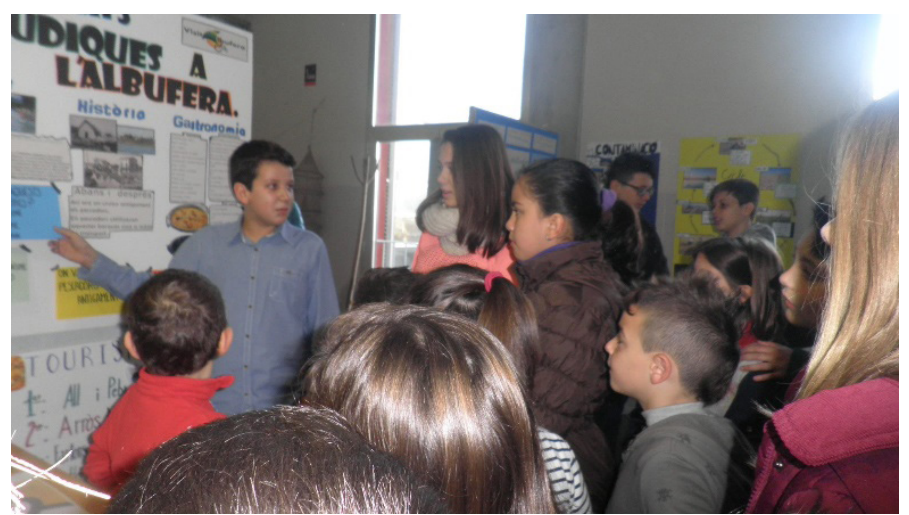

Fig. 3. Explicación de los guías al alumnado de primaria visitante.

\section{EVALUACIÓN Y CALIFICACIÓN DEL PROYECTO}

La evaluación del proyecto se establece desde el inicio como un instrumento de mejora del proceso de aprendizaje siguiendo los principios de la evaluación formadora definidos por Neus Sanmartí (2010). Se establecen momentos de recapitulación y de feedback entre alumnos y por parte de los docentes, con el fin de retroalimentar de un modo constructivo el proceso de aprendizaje que sigue cada alumno (Wiggins, 2012). Se trata de asegurar que en cada etapa del proyecto sea posible situarse dentro del itinerario completo. El alumnado debe poder responder a las preguntas: ¿Qué he aprendido hasta ahora? ¿Por dónde voy? ¿Qué me queda por aprender? Con este fin se establecen rúbricas de coevaluación para los productos expositivos que se evalúan de modo cruzado entre los diferentes grupos de expertos. También se evalúa el dosier de investigación y el dosier de experimentación del grupo de expertos con una rúbrica de autoevaluación revisada posteriormente por el equipo docente. El documento para recoger la información de la dinámica speed dating es un entregable individual que sirve como un instrumento más de evaluación. También se tiene en consideración para la evaluación la participación como guías de la exposición. Todos estos instrumentos tienen un peso específico para la calificación del alumnado en el proyecto.

Las asignaturas involucradas en el proyecto, Biología y Geografía, asignan un peso específico a la calificación del proyecto dentro de los instrumentos de calificación de la propia asignatura para cada alumno.

Tabla 5 .

Instrumentos de calificación y momentos de evaluación

\begin{tabular}{|c|l|l|}
\hline $\begin{array}{c}\text { Instrumento } \\
\text { de calificación }\end{array}$ & \multicolumn{1}{|c|}{ Modalidad } & \multicolumn{1}{|c|}{ Momento de evaluación } \\
\hline IG1 & $\begin{array}{l}\text { Dosier investigación grupal } \\
\text { Autoevaluación con rúbrica revisada por la/el docente }\end{array}$ & $\begin{array}{l}\text { Al final de la fase de trabajo en grupos } \\
\text { base }\end{array}$ \\
\hline II2 & $\begin{array}{l}\text { Dosier experimentación expertos } \\
\text { Autoevaluación con rúbrica revisada por la/el docente }\end{array}$ & Al final de la fase de expertos \\
\hline II3 & $\begin{array}{l}\text { Entregable resultado del speed dating } \\
\text { Evaluación por parte del/ de la docente }\end{array}$ & Después de la dinámica de speed dating \\
\hline IG4 & $\begin{array}{l}\text { Productos expositivos } \\
\text { Coevaluación entre grupos }\end{array}$ & $\begin{array}{l}\text { Tras el montaje definitivo del centro de } \\
\text { interpretación }\end{array}$ \\
\hline II5 & $\begin{array}{l}\text { Guía de exposición } \\
\text { Evaluación por parte del/de la docente }\end{array}$ & Tras la visita de los centros de primaria \\
\hline
\end{tabular}

*II- Instrumento individual - IG-Instrumento grupal 


\section{RESULTADOS}

Podemos diferenciar distintos tipos de resultados relacionados con la realización del proyecto expuesto:

- Respecto al cambio de metodología: se ha realizado la programación de cuatro horas semanales de todo un trimestre para segundo de la ESO en Ciencias Naturales y Ciencias Sociales a partir de una secuencia de actividades basada en los fundamentos del aprendizaje basado en retos.

- Respecto a la reflexión y trabajo en equipo del profesorado implicado: se ha creado un equipo estable de cuatro docentes que han diseñado, ejecutado y evaluado una secuencia didáctica en equipo.

- Respecto al alumnado: se diseñó una encuesta exprofeso para conocer la percepción del alumnado sobre el cambio producido. La encuesta se pasó a los tres grupos de segundo de la ESO (96 alumnos) al finalizar el proyecto y se obtuvieron los siguientes resultados:

Tabla 6.

Resultados de las encuestas pasadas al alumnado participante

\begin{tabular}{|l|l|}
\hline $\begin{array}{l}\text { El } 61,3 \% \text { de los alumnos considera que ha trabajado todo } \\
\text { lo que podía en el proyecto. }\end{array}$ & $\begin{array}{l}\text { El 19\% del alumnado considera que podría haber trabajado } \\
\text { más. }\end{array}$ \\
\hline $\begin{array}{l}\text { El } 34 \% \text { de los alumnos considera que han sido los com- } \\
\text { pañeros de grupo quienes más les han ayudado. }\end{array}$ & $\begin{array}{l}\text { El } 31 \% \text { del alumnado considera que quien más le ha ayuda- } \\
\text { do ha sido el docente. }\end{array}$ \\
\hline $\begin{array}{l}\text { La parte del proyecto que más ha gustado al alumnado ha } \\
\text { sido: al } 62,7 \% \text {, la salida a la Albufera; al } 16,2 \% \text {, el trabajo } \\
\text { en rol de expertos; al } 12,8 \% \text {, el trabajo de investigación en } \\
\text { grupos base, y al } 10,5 \%, \text { la sesión del speed dating. }\end{array}$ & \\
\hline
\end{tabular}

Se realizó también una encuesta en línea a las familias del alumnado con un índice de respuestas del $57 \%$ (tras tres envíos recordatorios durante las dos últimas semanas del proyecto), con los siguientes resultados destacados:

Tabla 7.

Resultados de las encuestas pasadas a las familias

El $81,4 \%$ de las familias considera que su hijo/a ha realizado una buena valoración del proyecto.

El $82,6 \%$ de las familias considera interesante $(37,2 \%)$ o muy interesante $(45,4 \%)$ que sus hijos aprendan ciencias naturales y sociales en la modalidad de proyecto.

El 87 \% de las familias afirma que sus hijos han compartido más información sobre el proyecto en casa que respecto a otras actividades del centro.

\section{CONCLUSIONES}

Retomando los objetivos que propiciaron la transformación de la «tradicional» salida de campo en un proyecto interdisciplinar que integrase el currículo de Biología y Geografía e Historia de segundo de la ESO, podemos constatar que dicha transformación fue llevada a cabo gracias a la coordinación y el trabajo en equipo de los docentes participantes. Es necesario destacar en este proceso la importancia de conocer las valoraciones previas sobre el trabajo desarrollado en las asignaturas implicadas para facilitar un impulso hacia el cambio a partir de actuaciones previas de éxito, en este caso la salida «tradicional». Es desde el propio conocimiento de la realidad del aula como pueden diseñarse mejoras que pivoten sobre las prácticas de éxito y no pretendan transformar completamente desde cero las actuaciones 
didácticas. El trabajo cotidiano de la profesión docente conlleva un ritmo de toma de decisiones y resolución de conflictos que dificulta la posibilidad de repensar las prácticas completamente. Si la transformación parte de una actuación valorada en positivo por parte del/ de la docente que la ejecuta, esta puede convertirse en la palanca que transmita la fuerza para generar el cambio.

En segundo lugar, cabe poner en valor la importancia del trabajo colaborativo del profesorado. Esta modalidad de codocencia ha generado un proceso de retroalimentación constante para el profesorado implicado, al compartir su experiencia y conocimientos durante el desarrollo de las clases diseñadas conjuntamente, siguiendo la línea de investigación de Rodríguez (2014). Y este hecho pone de manifiesto la relevancia de hacer posible la reflexión conjunta sobre la acción docente y de integrar diversas visiones al proceso de diseño y ejecución de una intervención didáctica. El codiseño de las secuencias didácticas conlleva un beneficio compartido para todo el equipo de docentes participantes en esta cocreación. Además, mejora en la calidad de la intervención didáctica y, por tanto, favorece el aprendizaje del alumnado.

La previsión de integrar en el horario de los docentes implicados un espacio para el encuentro programado para el curso completo es un elemento facilitador que permite generar sinergias de un modo estructurado. La reflexión didáctica compartida no puede realizarse como un extra ańadido al quehacer diario del cuerpo docente, sino en momentos específicos reservados para ello. Este hábito fomenta la colaboración, potencia la calidad de las actuaciones didácticas y propicia sinergias para la codocencia.

Los resultados, en cuanto a la percepción de los estudiantes sobre la transformación realizada, van en la línea de actuación que pretende mejorar lo que ponen de manifiesto diferentes estudios, como los de Solbes, Montserrat y Furió (2007), entre otros, sobre el bajo interés hacia las ciencias de los estudiantes de secundaria. Este cambio de actitud alcanzado es una evidencia de la necesidad de poner en práctica este tipo de planteamientos didácticos en el campo de la enseñanza de las ciencias (Osborne y Dillon, 2008). Además, esta valoración en positivo por parte del alumnado supone una buena predisposición, a priori, hacia nuevas actuaciones de estructura semejante que produzcan un efecto facilitador para que se den nuevos intentos.

Uno de los factores que dificultan los cambios en la metodología de trabajo en el aula suele ser la consideración de la necesidad de una mayor formación previa que asegure el éxito (Monereo, 2010); esta formación es necesaria, pero para conocer si un cambio está bien planteado es necesario ponerlo en práctica, evaluarlo y reflexionar sobre ello conjuntamente. Se debe comprobar que la resistencia es menor por parte del alumnado, la acogida es positiva por parte de las familias y la ejecución es formadora para el profesorado que la realiza en el desarrollo de nuevos roles docentes, como muestra el estudio de Valdés-Sánchez (2017).

Todos estos factores ayudan a reducir la percepción de dificultad para el cambio. Es desde la investigación-acción como puede mejorarse la realidad del aprendizaje de ciencias en el aula. Como ha evidenciado la ciencia a lo largo de su historia, cada paso es un avance.

Para concluir, es importante poner en evidencia las limitaciones de la actuación descrita: el contexto de cada centro es diferente y supone unas dificultades y/o facilidades intrínsecas para poder realizar diferentes tipos de intervenciones didácticas. Cada práctica debe surgir del conocimiento del propio contexto y desarrollarse a partir de los equipos docentes que vayan a ejecutarla. Con el fin de consolidar los beneficios encontrados en esta intervención, sería interesante analizar la evaluación del propio proyecto descrito a lo largo de otros cursos con nuevo alumnado y profesorado, así como transferir el proceso a un contexto diferente (otro centro educativo) y comprobar y comparar los resultados. 


\section{REFERENCIAS BIBLIOGRÁFICAS}

Aronson, E. y Bridgeman, D. (1996). The Jigsaw Technique. En Philip Banyard y Andrew Grayson, Introducing Psychological Research (pp. 65-68). https://doi.org/10.1007/978-1-349-24483-6_10

Beamish, W., Bryer, F. y Davies, M. (2006). Teacher Reflections on Co-Teaching a Unit of Work. International Journal of Whole Schooling, 2(2), 3-19.

Britner, S. L. y Pajares, F. (2006). Sources of science self-efficacy beliefs of middle school students. Journal of Research in Science Teaching: The Official Journal of the National Association for Research in Science Teaching, 43(5), 485-499. https://doi.org/10.1002/tea.20131

Cantó, J., Hurtado, A. y Vilches, A. (2013). Educación científica más allá del aula. Una herramienta para la formación del profesorado en sostenibilidad. Alambique, 74, 76-82.

Chanmugam, A. y Gerlach, B. (2013). A Co-Teaching Model for Developing Future Educators' Teaching Effectiveness. International Journal of Teaching and Learning in Higher Education, 25(1), 110-117.

Conderman, G. y Hedin, L. (2012). Purposeful assessment practices for co-teachers. Teaching Exceptional Children, 44(4), 18-27.

https://doi.org/10.1177\%2F004005991204400402

Cook, L. y Friend, M. (1995). Co-teaching: Guidelines for creating effective practices. Focus on Exceptional Children, 28. https://doi.org/10.17161/fec.v28i3.6852

Couso, D., Jiménez, M. P., López-Ruiz, J., Mans, C., Rodríguez, C., Rodríguez, J. M. y Sanmartí, N. (2011). Informe ENCIENDE: Enseñanza de las Ciencias en la Didáctica Escolar para Edades Tempranas en España. Madrid: COSCE.

Domenech-Casal, J. D. (2019). Aprenentatge basat en projectes, treballs pràctics i controvèrsies: 28 propostes $i$ reflexions per ensenyar ciències. Rosa Sensat.

Echevarría, T. Z., Alonso, J. S., González, G. M., Alonso, M. D. F. y Ugarte, I. E. (2014). Acercar la geodiversidad a través de las salidas de campo en la ESO. Una investigación con el profesorado de ciencias de Bizkaia. Enseñanza de las Ciencias: Revista de Investigación y Experiencias Didácticas, 32(3), 443-467.

http://dx.doi.org/10.5565/rev/ensciencias.1282

Larmer, J., Mergendoller, J. R. y Boss, S. (2015). Gold standard PBL: Essential project design elements. Buck Institute for Education, 1-4.

Monereo Font, C. (2010). ¡Saquen el libro de texto! Resistencia, obstáculos y alternativas en la formación de los docentes para el cambio educativo.

Osborne, J. y Dillon, J. (2008). Science education in Europe: Critical reflections (vol. 13). Londres: The Nuffield Foundation.

Pedrinaci, E. (2012). Alfabetización en Ciencias de la Tierra, una propuesta necesaria. Enseñanza de las Ciencias de la Tierra, 20(2), 133.

Pérez, A., De Pro, A. y Ato, M. (2005). Evaluación nacional de actitudes y valores hacia la ciencia en entornos educativos. Madrid: FECYT.

Rodríguez, F. (2014). La co-enseńanza una estrategia para el mejoramiento educativo y la inclusion. Revista Latinoamericana de Inclusión Educativa, 8(2), pp. 219-233.

Sanmartí, N. (2010). Avaluar per aprendre. L'avaluació per millorar els aprenentatges de l'alumnat en el marc del currículum per competències. Generalitat de Catalunya, Departament d'Educació, Direcció General de l'Educació Básica i el Batxillerat. 
Schunk, D. H. y Pajares, F. (2002). The development of academic self-efficacy. En Development of achievement motivation (pp. 15-31). Academic Press. https://doi.org/10.1016/B978-012750053-9/50003-6

Solbes, J., Montserrat, R. y Furió, C. (2007). Desinterés del alumnado hacia el aprendizaje de la ciencia: implicaciones en su enseñanza. Didáctica de las Ciencias Experimentales y Sociales, 21, 91-117.

Stuckey, M., Hofstein, A., Mamlok-Naaman, R. y Eilks, I. (2013). The meaning of 'relevance' in science education and its implications for the science curriculum. Studies in Science Education, $49(1), 1-34$.

https://doi.org/10.1080/03057267.2013.802463

Trujillo, F. (2016). ABP: Aprendizaje Basado en Proyectos (Secundaria y Bachillerato). Curso online de formación del profesorado. Ministerio de Educación, Cultura y Deporte/Instituto Nacional de Tecnologías Educativas y de Formación del Profesorado.

Valdés-Sánchez, L. (2017). La docencia compartida como herramienta de formación permanente de los maestros que integran ciencias e inglés. Enseñanza de las Ciencias, n. ${ }^{\circ}$ extra, 4943-4948.

Wiggins, G. (2012). Seven keys to effective feedback. Feedback, 70(1), 10-16.

Zavala, V. A. (2006). La práctica educativa. Como enseñar. México: Graó.

Zimmerman, B. J. (2000). Attaining self-regulation: A social cognitive perspective. En Handbook of self-regulation (pp. 13-39). Academic Press.

https://doi.org/10.1016/B978-012109890-2/50031-7 


\title{
$\ll$ We explain the Albufera $»$ : transforming a field trip into an interdisciplinary project
}

\author{
Enric Ortega-Torres \\ Unitat d’Educació, Florida Universitària, Catarroja (València) \\ eortega@florida-uni.es \\ Vicent Moncholí Pons \\ Departament de ciències, Florida Secundària, Catarroja (València) \\ vmoncholi@florida-uni.es
}

The teamwork shared by 4 secondary school teachers, two from natural sciences and two from social sciences, structured around one-hour meetings integrated into the schedule, planned and executed the project called «We explain the Albufera» that to date already has 6 successful editions.

The result of the didactic sequence which was designed was structured following the bases of the challenge-based learning methodology. Learning linked to a purpose that promotes the active participation of students through the need to use cognitive skills high level (HOTS) to achieve greater depth in learning.

The evaluation of the project is defined from the beginning as an instrument to improve the learning process following the principles of the formative evaluation. The aim is to ensure that at each stage of the project it is possible for students to answer the questions: What have I learned so far? Where am I going? What is left for me to learn?

There are different types of results:

- Regarding the change in methodology: the new four-hour-per-week sessions were planned in natural and social sciences defining a sequence of activities based on the fundamentals of challenge-based learning.

- Regarding the reflection and teacher's teamwork: a stable team of four teachers from different departments has been formed to design, execute, and evaluate a new learning sequence.

- Regarding the students: the perception of 96 students about the change produced was evaluated by means of a questionnaire.

The conclusions show the importance of collaborative work. The co-teaching modality exposed has generated a constant feedback process for the teachers involved, by sharing their experience and knowledge during the development of the project. This fact highlights the relevance of making possible joint reflection on teaching action and the importance of integrating different visions into the process of design and implementation of a didactic plan. The co-design of the didactic sequences entails a shared benefit for the entire team of teachers participating in this co-creation. In addition, it improves the quality of the didactic intervention and, therefore, the students' learning experience. 